\title{
Extension of Cosmic Noise Absorption Measurements to Lower Frequencies, Using Polarized Antennas
}

\author{
C. G. Little, ${ }^{1}$ G. M. Lerfald, ${ }^{1}$ and R. Parthasarathy ${ }^{2}$
}

(Received March 5, 1964; revised April 2, 1964)

\begin{abstract}
The cosmic-noise method of measuring absolute ionospheric absorption requires a reliable knowledge of the intensity of the cosmic radio noise which would be observed in the complete absence of the ionosphere. At frequencies above $15 \mathrm{Mc} / \mathrm{s}$ this extraterrestrial intensity has hitherto been estimated by a method which assumes that during ionospherically quiet, nighttime periods, the absorption becomes negligible. However, this "quiet-day" method leads to important uncertainties in the value of the extraterrestrial cosmic radio noise intensity when it is used at frequencies below about $15 \mathrm{Mc} / \mathrm{s}$.

This paper describes a technique, based on simultaneous measurements of the ordinary and extraordinary wave intensities, for obtaining the free-space intensity of the cosmic radio noise at frequencies as low as $5 \mathrm{Mc} / \mathrm{s}$. An experimental program is described which has verified the usefulness of the method and shown some of its limitations. The method is of value to studies of the spectrum of $\mathrm{HF}$ cosmic radio noise, and to the derivation of electron density profiles in the lower $D$-region using multifrequency absorption data.
\end{abstract}

\section{Introduction}

The measurement of ionospheric absorption utilizing observations of the intensity of cosmic radio noise incident on a ground-based antenna, was first used by Shain [1951]. The method has since been widely used at high latitudes [Little and Leinbach, 1958; Reid and Collins, 1959; Reid and Leinbach, 1959; Hultqvist, 1959; Stoffregen et al., 1960] as well as at lower latitudes (for example, see Steiger and Warwick [1961]). The antennas used for such observations have generally been combinations of dipoles arranged to receive linear polarization. In order to determine the net amount of absorption suffered by the radio energy in passing through the ionosphere, it is first necessary to estimate the intensity of the cosmic radio noise which would be observed if the ionosphere were absent. For a fixed receiving system, the latter intensity may be assumed to repeat exactly with a period of one sidereal day. The usual procedure for obtaining this sidereal variation of the cosmic noise has been one of observing for several weeks or months and then assuming that the highest interference-free signal levels observed at any given sidereal time represent times when there was negligible absorption. This method is satisfactory when the observing frequency is sufficiently high that there is indeed negligible absorption during a substantial fraction of the time. Since direct solar illumination of the $D$ region gives rise to appreciable absorption, nighttime observations are often necessary to obtain the sidereal variation of cosmic noise power by this method. Unfortunately, the relationship between

${ }_{1}$ Central Radio Propagation Laboratory, National Bureau of Standards, Boulder, Colo.

2 Geophysical Institute, University of Alaska, College, Alaska. the solar and sidereal days is such that at auroral latitudes certain sidereal hours never coincide with hours of darkness. Even at lower latitudes, it is usually necessary to observe for a full year in order to obtain nighttime conditions at all sidereal hours.

The determination of the sidereal day variation by the above method becomes increasingly uncertain as the frequency of observation is lowered. For example, at $30 \mathrm{Mc} / \mathrm{s}$ the "quiet-day" cosmic noise variation can be determined to an accuracy of perhaps $0.1 \mathrm{~dB}$; at $5 \mathrm{Mc} / \mathrm{s}$ the scatter in the daily values is so large that uncertainties of several decibels are encountered.

Benediktov [1959] has pointed out that for small values of absorption (less than about $2 \mathrm{~dB}$ ) it is theoretically possible to determine absorption values from a knowledge of the ordinary and extraordinary intensities without knowing the extraterrestrial cosmic noise intensity. A similar idea had occurred to staff members of the Geophysical Institute of the University of Alaska in 1955, and the method was used there by C. G. Little and the late Willis M. Rayton in early 1956 to check that the residual absorption at $30 \mathrm{Mc} / \mathrm{s}$ was indeed small.

This paper describes a technique which utilizes ground-based observations of the cosmic radio noise power in the ordinary and extraordinary polarizations to obtain reliable values of the sidereal day variation of the cosmic radio noise, and hence absorption values, at lower frequencies than are otherwise possible. The theoretical basis of the method is discussed and sample experimental results are presented from a program of dual-polarized measurements carried out at frequencies as low as $5 \mathrm{Mc} / \mathrm{s}$. The effects which tend to limit the accuracy of the method are also discussed. 


\section{Theory}

The existing evidence indicates that, at HF, the cosmic radio noise outside the earth's ionosphere is essentially randomly polarized when averaged over a broad beam antenna. However, on passing through an absorbing ionosphere, the extraordinary mode ( $X$-mode) signal will suffer more absorption than the ordinary mode $(O$-mode) signal. The quasi-longitudinal approximation of the classical AppletonHartree form of the magneto-ionic equation gives the relationship for the nondeviative absorption $A$, in decibels, for a plane wave propagating vertically at an effective operating frequency $f_{e}$ :

$$
A\left(f_{e}\right)=C \int_{h_{1}}^{h_{2}} \frac{N(h) \nu(h) d h}{\nu(h)^{2}+\left(2 \pi f_{e}\right)^{2}} .
$$

Here $C$ is a constant, $h$ is height above sea level, $N(h)$ is the electron density, and $\nu(h)$ is the collision frequency for electrons. The effective operating frequency, $f_{e}$, is given by the equation

$$
f_{e}=f \pm f_{L},
$$

where $f$ is the frequency to which the receiver is tuned and $f_{L}$ is the electron gyrofrequency for the longitudinal component of the magnetic field. The plus sign corresponds to the $O$-mode absorption and the minus sign to the $X$-mode absorption.

It may be seen from (1) and (2) that if a collision frequency profile which does not vary with time is assumed, the ratio of the absorption on the $O$ - and the $X$-modes at a given receiving frequency will be a function of the electron density profile. If the ionization occurs primarily at heights where $\nu>2 \pi f_{e}$, the absorption on the two modes will be approximately equal. On the other hand, if the absorption is occurring in a region where $\nu<<2 \pi f_{e}$ the ratio of the absorption on the two modes becomes

$$
\frac{A_{x}}{A_{o}}=\left(\frac{f_{e o}}{f_{e x}}\right)^{2}
$$

where $A_{o}$ and $A_{x}$ represent the absorption on the $O$ - and $X$-modes, respectively, and $f_{e o}$ and $f_{e x}$ are the corresponding effective operating frequencies. If the condition $\nu<<2 \pi f_{e}$ applies, (3) may be rewritten as

$$
\frac{A_{x}-A_{o}}{A_{o}}=\frac{f_{e o}^{2}}{f_{e x}^{2}}-1
$$

or for a given receiving frequency $f$

$$
\frac{A_{x}-A_{o}}{A_{o}}=S=\text { constant. }
$$

Let us assume that relative intensity measurements are made on circularly-polarized antennas at a given frequency and that $P_{0}$ and $P_{x}$ are the equivalent noise powers observed (in decibels above an arbitrary reference level) on the $O$ - and $X$-mode polarizations, respectively. The absorptions on the two polarizations are then defined by

and

$$
A_{o}=P-P_{0}
$$

$$
A_{x}=P-P_{x},
$$

where $P$ is the antenna noise power which would be observed on each polarization if the ionosphere were absent. Substituting in (5),

$$
P_{o}-P_{x}=S\left(P-P_{o}\right) \text {. }
$$

If it is assumed that $P$ is constant (which is true for a given sidereal time), a graph may be plotted of $P_{o}$ versus $P_{o}-P_{x}$ which, if (5) is valid, will define a line of constant slope. If the slope of this line is extended to the point where $P_{0}-P_{x}=0$, it may be seen from (7) that this intercept occurs at an intensity $P_{o}=P_{x}=P$. This intercept is therefore the noise power of the incident cosmic noise outside the ionosphere. Thus, at least in theory, simultaneous measurements of the ordinary and extraordinary wave noise powers can be used to deduce, by a simple extrapolation, the intensity of the cosmic radio noise which would be observed in the absence of the ionosphere.

The above discussion can be developed for the form of the magneto-ionic equation due to Sen and Wyller [1960] which takes account of the dependence of the effective electron collision frequency on the electron velocity distribution function. The results are very similar; the Appleton-Hartree form is used here because it permits the relationships between the ionospheric parameters to be shown more simply.

\section{Experimental Program}

The method outlined above has been used in the analysis of data collected at College, Alaska, during a 20-month observing period beginning in March 1961. The observational program involved measurements of the cosmic radio noise intensities incident on vertically directed antennas at frequencies of $5,10,20,30$, and $50 \mathrm{Mc} / \mathrm{s}$. Separate recordings of both the $O$ - and $X$-mode signals were made at the three lower frequencies while the 30 and $50 \mathrm{Mc} / \mathrm{s}$ systems had linearly polarized antennas. The antennas at all frequencies were designed to have identical beam patterns, each having circular symmetry about the zenith and a half-power response at $30 \mathrm{deg}$ off axis.

At frequencies of 30 and $50 \mathrm{Mc} / \mathrm{s}$, ionospheric absorption is usually not more than a few tenths of a decibel. The conventional "quiet-day" method was used to obtain estimates of the cosmic noise background by scaling the records and preparing a mass plot of relative power values versus local sidereal time. Figure 1 shows such a plot for the $50 \mathrm{Mc} / \mathrm{s}$ observations during the month of February 1962. The upper envelope is seen to be fairly well defined and can be used to estimate the cosmic 
noise variation with an uncertainty of perhaps $0.2 \mathrm{~dB}$. (The addition of data taken in other months permits the reduction of this uncertainty to about $0.1 \mathrm{~dB}$.) On the other hand, in accordance with the approximate inverse-frequency-squared variation of absorption, the lower frequencies demonstrated the presence of appreciable absorption much of the time. For example, see figure 2, which shows a mass plot of $10 \mathrm{Mc} / \mathrm{s} O$-mode relative power values versus sidereal time. The greatly increased scatter of points in figure 2 is due to the occurrence of appreciable absorption much of the time, and prevents the derivation of an accurate quiet-day curve by the mass-plot method.

Figure 3 shows plots of the $O$-mode antenna power, $P_{o}$, versus the difference between the $O$ - and $X$-mode powers, $P_{0}-P_{x}$, for the points in figure 2 which lie within the specified sidereal hourly intervals. A regression line fitted to the points on each graph was then used to estimate the most probable value of the intensity of the cosmic radio noise at the sidereal hour in question. (In practice, a similar plot was prepared for each sidereal hourly period.) The slopes of the regression lines in $a$ and $c$ of figure 3 are representative of most hours and are in fact those expected from a uniform layer of ionization occurring principally at heights greater than about $70 \mathrm{~km}$. On the other hand, the slope of the regression line in $3 b$ is significantly different and is representative of one of the more extreme departures from the average. However, the slope in the latter case is controlled by a few points from absorption events which were found to deviate from the square-law condition of (3). The possible causes of such differences in the data will be discussed in sections $4.1 \mathrm{c}$ and $4.2 \mathrm{c}$ of this paper. It is seen from figure 3 , that the intercept of the regression line with $P_{0}-P_{x}=0$ is not shifted greatly by the changes of slope which have been encountered. The repeatability of the results of the determinations is indicated by figure 4 , which shows


Figure 1. Mass plot of $50 \mathrm{Mc} / \mathrm{s}$ hourly scalings for month of February 1962 versus local sidereal time.

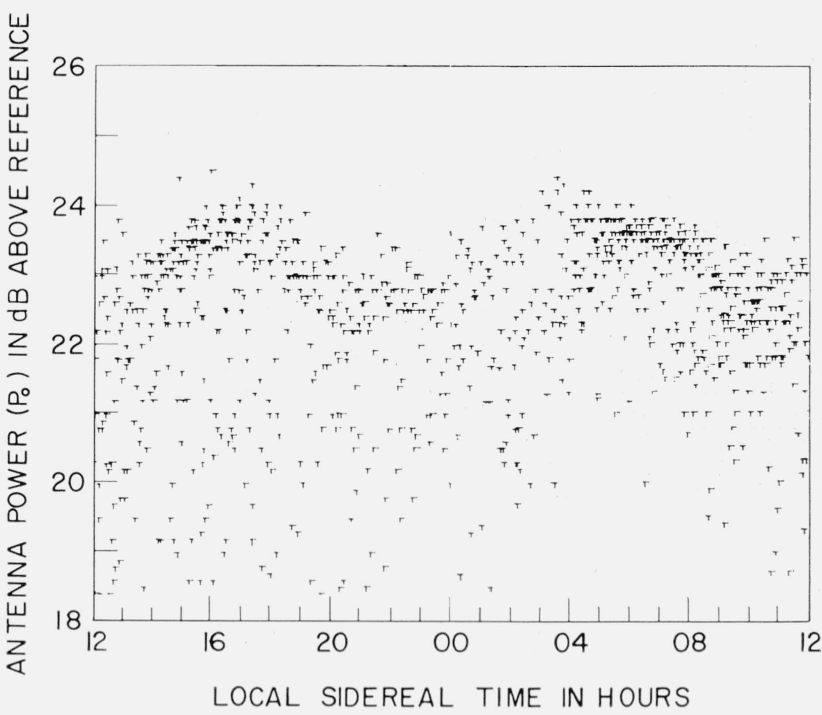

Figure 2. Mass plot of $10 \mathrm{Mc} / \mathrm{s}$ hourly O-mode scalings for month of February 1962 versus local sidereal time.

The vertical scale, like that of figure 1 , covers $8 \mathrm{~dB}$. The greater incidence of absorption (compared to that observed at $50 \mathrm{Mc} / \mathrm{s}$ ) is to be expected in view of the approximate inverse-frequency-squared relationship of absorption.



Figure 3. Plots of $10 \mathrm{Mc} / \mathrm{s}$ O-mode relative antenna power versus the differential in the $O$ - and $X$-mode polarized antenna powers for selected hourly periods of sidereal time using data from the month of February 1962.

A best-fit regression line is used to estimate the relative antenna power which would be observed in the absence of an absorbing ionosphere. The slopes of the regression lines are indicative of the absorption-frequency relationship during events.

the sidereal time variation obtained using three groups of $10 \mathrm{Mc} / \mathrm{s}$ data, each of one month duration. A smoothed curve is fitted to the experimentally determined values (heavy dashed line) and lines are drawn $0.25 \mathrm{~dB}$ above and below the latter. Nearly all the points fall within these limits.

The individual datum points at $10 \mathrm{Mc} / \mathrm{s}$ have an uncertainty of about $\pm 0.2 \mathrm{~dB}$ due to scaling errors 
and this accounts for some, but not all, of the deviation from the regression lines shown in figure 3 . The following sections will discuss the other causes of this scattering.

The $5 \mathrm{Mc} / \mathrm{s}$ data were handled the same way as the $10 \mathrm{Mc} / \mathrm{s}$ information. As examples, values of $P_{o}$ versus $P_{o}-P_{x}$ obtained between 04:00 and 04:59, local sidereal time in January and February 1962, are plotted on figures $5 \mathrm{a}$ and $5 \mathrm{~b}$, respectively. In comparison with the $10 \mathrm{Mc} / \mathrm{s}$ data, increased absorption activity is evidenced. However, it appears that a reasonably good estimate (within about $\pm 0.5 \mathrm{~dB}$ ) of the extraterrestrial noise power is obtained by the regression line fit.



Figure 4. Sidereal time variation of cosmic radio noise at $10 \mathrm{Mc} / \mathrm{s}$ determined by monthly groups of data, viz, scalings from January, February, and March 1962.

A curve which represents the 3 hour running mean of the average of the hourly values is shown, as well as curves which are $0.25 \mathrm{~dB}$ above and below the latter (light dashed lines).
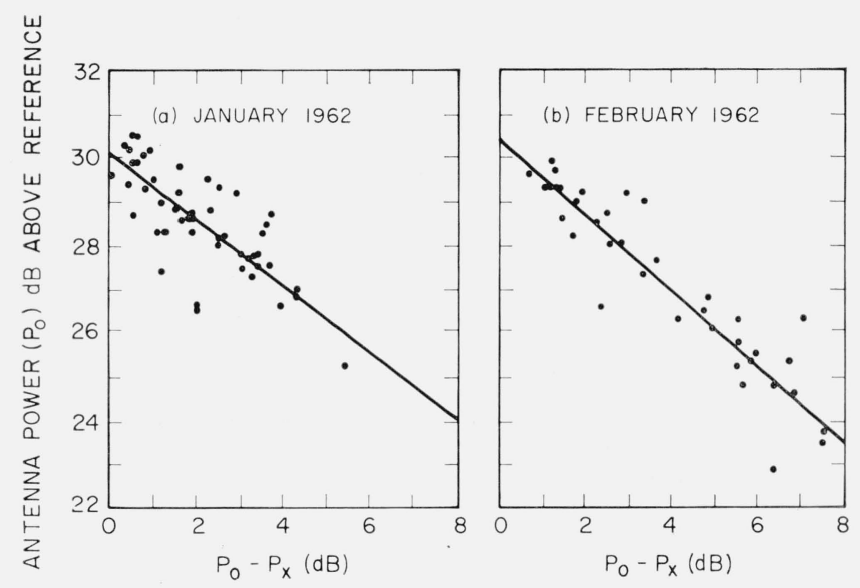

Figure 5. Scatter plots of $\mathrm{P}_{\mathrm{o}}$ versus $\mathrm{P}_{\mathrm{o}}-\mathrm{P}_{\mathrm{x}}$ at $5 \mathrm{Mc} / \mathrm{s}$ for the hourly sidereal period, 04:00-04:59, for (a) January 1962 and (b) February 1962.

\section{Problems and Limitations}

It is evident, particularly at $5 \mathrm{Mc} / \mathrm{s}$ that individual datum points on cross plots of $P_{o}$ versus $P_{o}-P_{x}$ exhibit more scatter about the regression line than can be explained by scaling uncertainties alone. Other factors which can give rise to scatter will be arbitrarily separated into those which are primarily due to the use of broad beamwidth antennas and those which are not. The former class of factors will be discussed first.

\subsection{Effects Due to the Use of Broad Beamwidth Antennas \\ a. General}

In the following discussion we assume a polar coordinate system with an antenna located at the origin, using $\theta$, the angle measured from the zenith, and $\phi$, the angle measured eastward from geographic north (azimuth angle). In the case of a broad beam antenna located above a perfectly reflecting ground screen, the power produced at the output terminals can be expressed as

$$
P\left(f_{e}\right)=C_{1} \int_{\theta=0}^{\theta=\frac{\pi}{2}} \int_{\phi=0}^{\phi=2 \pi} G(\theta, \phi) T(\theta, \phi) \sin \theta d \theta d \phi,
$$

where $C_{1}$ is a constant, $G(\theta, \phi)$ is the power gain of the broad beam antenna as a function of direction, and $T(\theta, \phi)$ is the equivalent noise temperature viewed from the antenna.

If $T(\theta, \phi)$ is expressed as equivalent source temperature in decibels above a reference temperature, $T_{R}$, its magnitude is given by

$$
T(\theta, \phi)=T_{S}(\theta, \phi)-A(\theta, \phi),
$$

where $T_{s}(\theta, \phi)$ is the true sky temperature (the temperature which would be derived in the absence of the ionosphere) in decibels above $T_{R}$ and $A(\theta, \phi)$ is the total integrated absorption, in decibels, along the propagation path. Losses in the antenna and the temperature contribution of the absorbing layer are neglected here since they become important only in the case of large absorption values. We shall examine, in turn, the limitations imposed on the technique outlined in section 2 of this paper by (1) nonuniformity of the sky background temperature, $T_{S}(\theta, \phi),(2)$ nonuniformity of the absorption layer in the horizontal extent, and (3) the effect of leakage between modes of the circularly-polarized antennas.

\section{b. Non-Uniformity of Sky Background}

If the ionosphere were completely absent, a vertically directed antenna on earth would register a variation in the cosmic noise equivalent temperature which would repeat exactly with a period of one 
sidereal day. At all the frequencies used in the experiment at College (lat. $65^{\circ} \mathrm{N}$.), the maximum and minimum sky temperatures during the course of a sidereal day were found to differ by less than $3 \mathrm{~dB}$, and the maximum rate of change per sidereal hour was about $0.4 \mathrm{~dB} / \mathrm{hr}$. Since the dual-polarized data were grouped into hourly intervals, the cosmic noise background integrated over the antenna beam, could vary by $\pm 0.2 \mathrm{~dB}$ according to whether the reading was performed near the beginning or end of the sidereal hour. Thus a scatter of this magnitude in the individual datum points is introduced by the varying sky temperature during portions of the sidereal day when the background is changing most rapidly. However, the records were scaled in local meridian time intervals so that the sidereal time of the readings was shifted by $4 \mathrm{~min}$ per day. The result was that a month's values were evenly distributed in sidereal time so that an average value could be used for each hour. Thus it has not been necessary to break the data into periods shorter than one sidereal hour and the nonuniformity of the cosmic noise background does not contribute significantly to errors in the broad beam cosmic noise temperature determinations.

\section{c. Non-Uniformity of Absorption in Horizontal Extent}

The following example serves to demonstrate that non-uniform distributions of ionization across the pattern of a broad beam antenna can result in variations in the absorption-frequency relationship. If one assumes that 20 percent of the effective beam pattern is covered with a patch of intense ionization which is optically thick for both the $O_{-}$and $X$ modes at the observing frequency concerned, and that the remainder of the beam does not intercept an absorbing region, then about $1 \mathrm{~dB}$ of absorption would be observed on both modes. Thus the $P_{o}$ value on a $P_{o}$ versus $P_{0}-P_{x}$ plot (e.g., figs. 3 or 5 ) would be shifted downward while $P_{0}-P_{x}$ would remain the same, resulting in a datum point which deviated from those obtained during times when a uniform layer of ionization was present.

The example cited is believed to be more extreme than commonly occurs at the auroral zone. For example, from studies employing spaced riometers [Holt, Landmark, and Lied, 1961; Little, Schiffmacher, Chivers, and Sullivan [private communication], it is known that many auroral absorption events correlate reasonably well at spacings greater than $100 \mathrm{~km}$. (Typically, the correlation coefficient drops to 0.5 at about $500 \mathrm{~km}$ spacing.) Also, absorption measurements made at Saskatoon using a relatively narrow beam antenna $( \pm 6 \mathrm{deg})$ which was alternately directed $12 \mathrm{deg}$ north and south of the zenith [Kavadas, 1961], have indicated good uniformity for most events. These results imply that only small deviations from horizontal uniformity could occur over a $\pm 30^{\circ}$ antenna beam. However, other evidence reported by Ansari [1963] indicates the occasional occurrence of events which exhibit areas of absorption a few kilometers or tens of kilometers in extent. Thus, it appears that scattered datum points could result from localized absorption patches, but it is difficult to quantitatively estimate the fraction which might be seriously affected in this way.

\section{d. Leakage Between Polarized Antenna Outputs}

A circularly-polarized antenna of finite beamwidth picks up some signal on the opposite mode, and this appears at the output and constitutes a contamination of the desired polarization. This contamination becomes quite important during intense absorption events when the difference between the $O$ - and $X$-mode temperatures may become very large. With a knowledge of the details of the antenna beam pattern, corrections may be readily computed. Such corrections were made on some of the data to determine the effect on the zerodifferential value, and it was found that the application of the leakage correction resulted in only negligibly small $(<0.05 \mathrm{~dB})$ changes in the value.

\subsection{Effects Not Primarily Related to Antenna Beamwidth Characteristics}

\section{a. Interference}

Interference due to transmitters and atmospheric noise was encountered at times, particularly at 5 $\mathrm{Mc} / \mathrm{s}$. However, the presence of interference of these types was usually apparent from the appearance of the records (due to the characteristic time variations of the interference); periods which showed any trace of such interference were not used. The possibility that interference with the spectral characteristics of random noise might have occurred occasionally, cannot be ruled out. Solar radio noise is of this type, as well as noise generated by synchrotron emission from energetic electrons spiraling in the terrestrial magnetic field. Interference due to solar radio noise was occasionally observed but generally occurred in bursts lasting only a few tens of minutes and was therefore recognizable. No evidence indicating serious contamination by longer duration, Type IV, solar-noise storms was noted. This may be partially attributable to the fact that, on an annual basis, the solar zenith angle at College, is greater than $60 \mathrm{deg}$ almost 90 percent of the time; the computed antenna gain at such angles is more than $13 \mathrm{~dB}$ down from that in the zenith. Noise generated by the synchrotron process would be likely to occur most often during disturbed periods when absorption was present and could result in a scattering of the points. However, from a study of the absorption-frequency relationship of data collected simultaneously at various frequencies in the range, 5 to $50 \mathrm{Mc} / \mathrm{s}$, it appears that instances of serious contamination by undetected interference are relatively rare. Hence, it is believed that the most serious effect of interference is the resulting loss of data necessitating longer periods of operation to obtain a given level of statistical significance. 


\section{b. $E$ - and $F$-Region Deviative Effects}

Cosmic-noise observations on broad beam antennas are subject to deviative effects which become noticeable when the critical frequency is such that the ionosphere begins to limit the area of sky viewed by the antenna (e.g., see Steiger and Warwick [1961]). In order to minimize this effect, the antennas used for the present experiment were specifically designed to have low sensitivity at high zenith angles. In practice, the antenna power gain for angles near the horizon, is expected to be no more than 3 percent of the zenithal response. Computations indicate that for an observing frequency of $10 \mathrm{Mc} / \mathrm{s}$, deviative effects are negligible for critical frequencies less than $3.5 \mathrm{Mc} / \mathrm{s}$, will result in a slight underestimation $(<0.25 \mathrm{~dB})$ of the derived sky temperatures for critical frequencies ranging from $3.5-5.0 \mathrm{Mc} / \mathrm{s}$ and will result in an overestimation of the sky temperature for critical frequencies greater than $6.0 \mathrm{Mc} / \mathrm{s}$.

The critical frequencies at College, Alaska, during the observing period remained mostly in the range 4.0 to $5.5 \mathrm{Mc} / \mathrm{s}$ when the $F$ region was illuminated and considerably less at night. Under these conditions the $P_{0}-P_{x}=0$ intercept on plots such as figure 3 should not be influenced greatly by deviative effects. This viewpoint was borne out by a test in which $P_{o}-P_{x}=0$ intercepts were determined using $10 \mathrm{Mc} / \mathrm{s}$ data which were (a) unscreened, and (b) screened by rejecting data taken when $f_{0} F 2$ was greater than $4.0 \mathrm{Mc} / \mathrm{s}$; the intercepts obtained using the two sets of data in this way were not significantly different.

At $5 \mathrm{Mc} / \mathrm{s}$, deviative effects limit the useful data to that taken during winter (1 October to $30 \mathrm{March}$ ), nighttime periods when the critical frequencies are low. The effect of the change in $F$-region critical frequencies near sunrise and sunset during winter is usually clearly evident on the $5 \mathrm{Mc} / \mathrm{s}$ records and can be used to reject periods affected by deviative

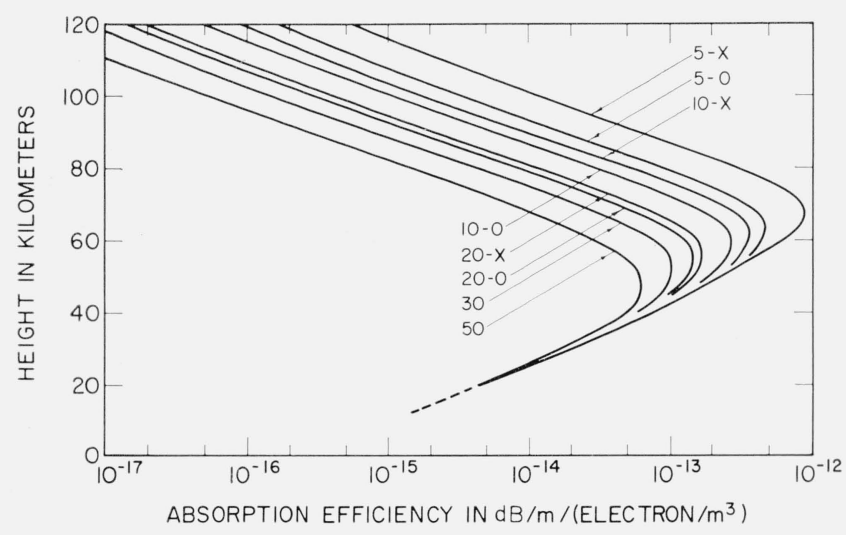

FiguRe 6. Height profiles of absorption in decibels per unit path length at height $\mathrm{h}$ for electron density $\mathrm{N}=1$ for a number of different radio-wave frequencies and polarizations.

The numbers adjacent to the curves represent the frequency, while the $O$ and $X$ designate the ordinary and extraordinary mode, respectively. absorption. Another obvious way of detecting data influenced by deviative absorption is by reference to ionograms taken simultaneously. The datum points shown on figure 5 were those remaining after rejection of data taken when the $F$-region critical frequency was observed to be greater than $2.5 \mathrm{Mc} / \mathrm{s}$. It is estimated that a critical frequency of $2.5 \mathrm{Mc} / \mathrm{s}$ will result in a shift of no more than $0.5 \mathrm{~dB}$ in the value of an individual datum point on figure 5 . Unfortunately, critical frequency values were not obtained during a substantial fraction of the time because of ionospheric blackout conditions and sounder malfunction.

\section{c. Ionization Below $80 \mathrm{~km}$}

The total nondeviative absorption, in decibels, experienced by a radio wave arriving from the zenith may be expressed by

$$
A\left(f_{e}\right)=\int_{0}^{\infty} K\left(f_{e}, h\right) \cdot N(h) d h,
$$

where

$A\left(f_{e}\right)=$ absorption in decibels at frequency $f_{e}$,

$K\left(f_{e}, h\right)=$ absorption in decibels per unit path length at height $h$ for electron density $N=1$ at frequency $f_{e}$,

$N(h)=$ the number of free electrons per unit volume as a function of height.

Values of $K\left(f_{e}, h\right)$ for a number of frequencies were computed using the Sen-Wyller form of the magnetoionic equations and the profile of electron collision frequency, $\nu(h)$, given by Nicolet [1959] and are shown in figure 6 . The peak value of each of the $K(h)$ curves occurs at a height where $\nu \approx 2 \pi f_{e}$. If all of the ionization giving rise to radio wave absorption occurs at heights where $\nu<<2 \pi f_{e}$ (i.e., at heights greater than $80 \mathrm{~km}$ for $f \geq 5 \mathrm{Mc} / \mathrm{s}$,) the inverse frequency squared relationship of absorption will obtain and the condition of (3) will be satisfied. If, on the other hand, the ionization-height profile varies in such a manner that, at different times, differing fractions of the absorption occur at heights where $\nu$ is comparable to or greater than $2 \pi f_{e}$, the absorption-frequency relationship will vary and the result will be an increased scattering of points on $P_{o}$ versus $P_{o}-P_{x}$ plots.

During the polar cap absorption events of July 1220, 1961, such changes in the slope were observed during various stages of the events. Observations at other stations indicated that reasonably good uniformity of absorption across the antenna beam pattern could be assumed and the changes were therefore attributed to variations in the electrondensity height-profile below $80 \mathrm{~km}$. These data have been used to derive the electron-density profiles for these polar cap absorption events [Parthasarathy, Lerfald, and Little, 1963]. 


\section{Conclusions}

Simultaneous measurements of the cosmic noise power on both circular polarizations have been used at 5, 10, and $20 \mathrm{Mc} / \mathrm{s}$ to derive the extraterrestrial noise power at these frequencies. The method has been found to be a considerable improvement over the standard "quiet-day curve" method commonly used; it also has the considerable advantage that the sidereal variation of noise power can be obtained in periods of the order of days instead of many months.

Various effects which might produce errors in determinations of the extraterrestrial cosmic radio noise power by this method, have been described in detail. It is seen that certain of these perturbing influences (viz, non-uniformity of sky background temperature and leakage between polarization modes) give rise to effects which are comparable to or smaller than the normal measuring and scaling uncertainties, whereas others (viz, interference and deviative absorption effects) can, under most conditions, be reduced to reasonably small values by suitably screening the data.

It has been shown that varying degrees of nonuniformity of ionization in the horizontal plane, and varying amounts of low lying ionization, will result in variations of the value of $S$ in (7). Such fluctuations will therefore lead to a scattering of the datum points from a line of constant slope (e.g., see figs. 3 and 5). An analysis of the observed frequency dependence of absorption suggests that about half of the $5 \mathrm{Mc} / \mathrm{s}$ points are significantly affected by deviation from the $1 / f^{2}$ law; it has not been possible using the multifrequency, dual-polarized data to identify whether horizontal nonuniformity or low lying ionization was the principal source of deviation from the $1 / f^{2}$ law.

It has been seen that at $5 \mathrm{Mc} / \mathrm{s}$, deviative absorption effects limit the observing time to winter nighttime periods. The extension of the method to lower frequencies is likely to be limited by deviative absorption effects in the $E$ and $F$ regions, and this would ultimately reduce the observing time to zero. When ionosonde recordings are available they permit a quantitative evaluation of deviative effects, and so aid greatly the selection of data and might conceivably be used to correct the observations in marginal cases.

The effects of horizontal nonuniformities of the ionization could be alleviated by the use of much narrower beam antennas (an economically unattractive solution), or the use of a network of absorption equipments spaced by about $50 \mathrm{~km}$. With the latter system, it would be possible to differentiate between departures from the $1 / f^{2}$ law due to horizontal nonuniformities and those due to violation of the condition $\nu<<2 \pi f_{e}$.

The method outlined in this paper has permitted improved accuracy in determining the cosmic noise equivalent temperatures, which would be observed at frequencies below $20 \mathrm{Mc} / \mathrm{s}$, if the ionosphere were absent. With a knowledge of the latter, reliable values of absolute absorption can be computed; such absorption data have been utilized to obtain electrondensity profiles in the $30-80 \mathrm{~km}$ height range by a method described in a previous paper [Parthasarathy, Little, and Lerfald, 1963].

The cosmic noise temperatures are of considerable interest in themselves because of the inferences which can be made concerning the generation of the cosmic radio energy and its propagation through interstellar space.

This work was supported in part by the Advanced Research Projects Agency, Nuclear Test Detection Office and was monitored by Major H. Dickinson under Contract 183-62.

\section{References}

Ansari, Z. A. (1963), The spatial and temporal variations in high latitude cosmic noise absorption and their relation to luminous aurora, University of Alaska Geophysical Institute Report UAG R-138.

Benediktov, E. A. (1959), On a radioastronomical method for determination of the absorption of radio waves in the ionosphere, Radiotechn. i Elektron. 4, No. 7, 1201-1202.

Holt, O., B. Landmark, and F. Lied (1961), Analysis of riometer observations obtained during polar radio blackouts, J. Atmospheric Terrest. Phys. 23, 229-243.

Hultqvist, B. (1959), On the interpretation of ionization in the lower ionosphere occurring on both day and night side of the earth a few hours after some solar flares, Tellus 11, No. 3, 332-343.

Kavadas, A. (1961), Absorption measurements near the auroral zone, J. Atmospheric Terrest. Phys. 23, 170-176.

Little, C. G., and H. Leinbach (1958), Some mesaurements of high-latitude ionospheric absorption using extraterrestrial radio waves, Proc. IRE 46, 334-348.

Nicolet, M. (1959), Constitution of the atmosphere at ionospheric levels, J. Geophys. Res. 64, No. 12, 2092-2101.

Parthasarathy, R., G. M. Lerfald, and C. G. Little (1963), Derivation of electron-density profiles in the lower ionosphere using radio absorption measurements at multiple frequencies, J. Geophys. Res. 68, No. 12, 3581-3588.

Reid, G. C., and C. Collins (1959), Observations of abnormal vhf radio wave absorption at medium and high latitudes, J. Atmospheric Terrest. Phys. 14, Nos. 1 and 2, 63-81.

Reid, G. C., and H. Leinbach (1959), Low-energy cosmic ray events associated with solar flares, J. Geophys. Res. 64, 1801-1805.

Sen, H. K., and A. A. Wyller (1960), On the generalization. of the Appleton-Hartree magnetoionic formulas, J. Geophys. Res. 65, No. 12, 3931-3950.

Shain, C. A. (1951), Galactic radiation at $18.3 \mathrm{Mc} / \mathrm{s}$, Australian J. of Scientific Res., Series A 4, No. 3, 258-267.

Steiger, W. R., and J. W. Warwick (1961), Observations of cosmic radio noise at $18 \mathrm{Mc} / \mathrm{s}$ in Hawaii, J. Geophys. Res. 66, No. 1, 57-66.

Stoffregen, W., H. Derblom, and A. Omholt (1960), Some characteristics of the $D$-region ionoization during auroral activity, J. Geophys. Res. 65, No. 6, 1699-1704.

(Paper 68D8-384) 